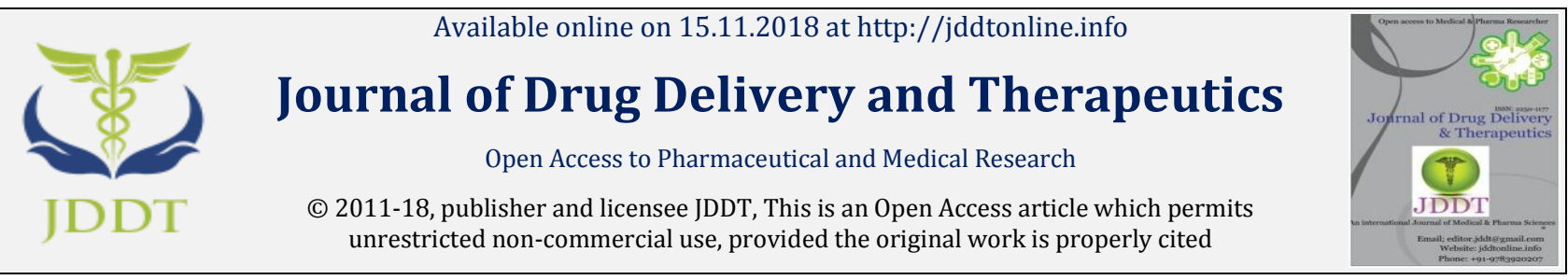

Open $\odot$ Access

Mini Review

\title{
Mini-Review: Nanotechnology Forms of Drug Delivery
}

\author{
Xingde Zhang 1, 2, 3 \\ ${ }^{1}$ School of pharmacy, Nanjing University of Chinese Medicine, Nanjing 210023, P. R. China \\ ${ }^{2}$ State Key Laboratory Cultivation Base for TCM Quality and Efficacy, Nanjing University of Chinese Medicine, Nanjing 210023, P. R. China \\ 3 Yancheng Traditional Chinese Medicine Hospital Affiliated to Nanjing University of Chinese Medicine
}

\begin{abstract}
The work seeks to present nanotechnology forms of drug delivery and how it would improve treatment effects to patients. Nanotechnology forms of drug delivery not only offer many options for cancer patients using chemotherapeutic drugs, but it also has much more alternative uses and this review will seek to present data on the same.
\end{abstract}

Keywords: Nanotechnology, covalent conjugation, non-covalent encapsulation

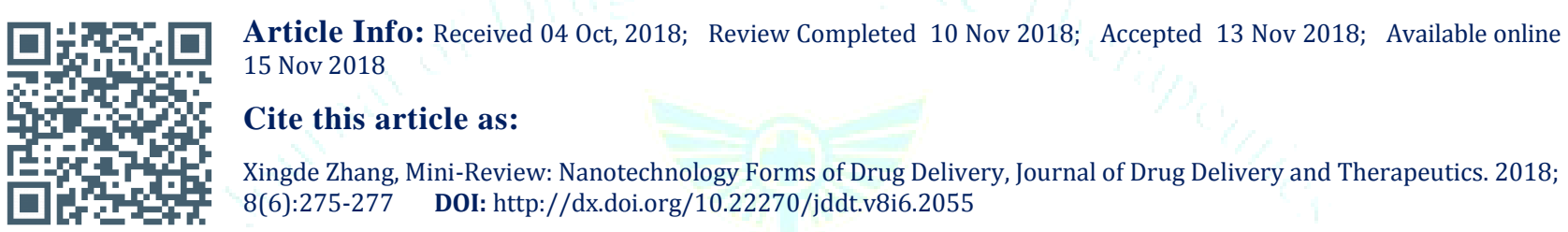

\section{Introduction}

In the treatment of cancer, different forms of chemotherapeutic drugs are used. The drugs have a significant advantage for patient therapy but they have some disadvantages as well. They are toxic. They have poor tissue selectivity. They have a narrow therapeutic window and the continued use of such drugs would lead to drug resistance ${ }^{1-9}$. Toxicity is related to the action of drugs on all cells (the cancer cells as well as healthy tissue cells) which would result in the death of the healthy tissue cells. A selectively targeted drug delivery system is needed to improve the prognosis for the patient. This is done with the help of nanotechnology. With nanotechnology, it is possible to prepare nanoparticles with different size, shapes, chemical, and physical properties $10-16$. Due to their unique size, the range of nanoparticles exhibit and more, an "enhanced permeability and retention effect" (EPR) is observed. This helps the drugs to specifically target and accumulate in the tumor cells. The work reviews the nanotechnology implementation of drug delivery and its efficacy with the help of existing secondary research studies.

\section{Nanotechnology forms of delivery}

Two ways to load the drug in the delivery vehicle in nanotechnology forms of delivery are by means of noncovalent encapsulation and covalent conjugation. In noncovalent encapsulation, the drug molecules interact with the delivery materials by non-covalent bonding, which offers advantages of adjusting the ratio of dose and multidrug combination. However, the weak non-covalent nature would normally lead to instability in drug releases ${ }^{17-23}$.

In covalent conjugation, both the drugs and the delivery materials are chemically bonded together, enabling the delivery vehicle as a predictable profile. This is a necessity in all treatment plans. The Captothecin CPT, which is derived from Chinese tree Camptotheca, exhibits very strong cytotoxicity and hence it offers much value for cancer treatment. As tested in vivo and in vitro, it is identified that CPT is a DNA topoisomerase inhibitor. The efficacy of CPT is primarily due to the formation of the tertiary complex between topoisomerase, DNA and CPT. Rapid accumulation of Cancer DNA is thus terminated. Some issues are noticed in the treatment delivery. However, for instance, the instability of the e-lactone ring in CPT leads to the formation of carboxylate forms that are inactive. CPT derivates approved by the FDA are helpful for the treatment of cancers of the ovary and colon cancer as well. There are pros of using CPT as CPT has a 5-membered ring system and this system makes the compound better in hydrophobic delivery ${ }^{24-32}$.

The advantages of the CPT make it a model compound to be used in drug delivery. Especially in the case of hydrophobic drug delivery, it is identified that nanoparticles in their simple assembly need a hydrophilic structure and, in this $\mathrm{CPT}$, it can be used. Natural amino acids and peptides structures are used for drug delivery. They do have biocompatibility and also reduced systemic toxicity. Ethylene glycol is used as a hydrophilic part as well. In selfassembly of polymers, disulfide bonding is used for the purpose of cross-linking. Stability is always an issue in drug 
delivery and in the use of nanotechnology with some of these natural peptide structures, it has been identified that stability is corrected to a certain extent. A CPT conjugated cross-linked micelles structure with built-in the disulfide bond, for instance, can be broken down faster into the bloodstream. It diffuses faster into the bloodstream.

With drug resistance and other serious issues happening in traditional treatment methods, the use of nanotechnology can aid in better imparting of multiple drug treatment to patients. The use of nanotechnology helps address the issues of cytotoxicity happening in cancer drug treatments. In the case of aggressive cancer, it is necessary to load much medicine as a single delivery option in order to slow down the rapid abnormal proliferation ${ }^{33-36}$. However, this form of medicine loading, in turn, does damage to the person's health as their normal cells and tissues are targeted as well. The efficacy of the chemotherapeutic drugs hence has to be re-analyzed. In this context, nanotechnology delivery forms become important.

Additionally, as Yang et al. and Sun et al. highlight, nanotechnology does need to be useful in certain medical

\section{References}

1. Qiao, H., et al. Redox-triggered mitoxantrone prodrug micelles for overcoming multidrug-resistant breast cancer. Journal of drug targeting 2018; 26:75-85.

2. Kang, C., Qin, J., Osei, W. \& Hu, K. Regulation of protein kinase $\mathrm{C}$-epsilon and its age-dependence. Biochemical and Biophysical Research Communications 2017; 482:1201-1206).

3. Sun, Y., et al. RGD Peptide-Based Target Drug Delivery of Doxorubicin Nanomedicine. Drug development research 2017; 78:283-291.

4. Kang, C. \& Hu, K. Role of caveolin-3 in adenosine-induced increase in mitochondrial PKCE. The FASEB Journal 2013; 27:1191.1197-1191.1197.

5. Cheng, X. \& Lee, R.J. The role of helper lipids in lipid nanoparticles (LNPs) designed for oligonucleotide delivery. Adv Drug Deliv Rev 2016; 99:129-137.

6. Sun, Y. \& Kang, C. Self-Assembly of Peptides into Hydrogel. Journal of Organic \& Inorganic Chemistry 2016; 2:5.

7. Yao, Z., Sun, Y. \& Kang, C. Structure and self-assembly of multicolored Naphthalene Diimides Semiconductor. Nano LIFE 2016; 6:1642007.

8. Cheng, X., et al. T7 Peptide-Conjugated Lipid Nanoparticles for Dual Modulation of Bcl-2 and Akt-1 in Lung and Cervical Carcinomas. Molecular pharmaceutics 2018; 15:4722-4732.

9. Zhong, X., Sun, Y., Kang, C. \& Wan, G. The theory of dielectrophoresis and its applications on medical and materials research. European Journal of BioMedical Research 2017; 2:7-11.

10. Fan, S. \& Chi, W. Methods for genome-wide DNA methylation analysis in human cancer. Brief Funct Genomics 2016; 15:432442.

11. Kang, C. \& Hu, K. Modulation of the two-pore domain potassium channel TASK-1 by caveolin-3. The FASEB Journal 2015; 29:845.814.

12. Kang, C., Sun, Y., Wang, M. \& Cheng, X. Nanosized camptothecin conjugates for single and combined drug delivery. European Journal of BioMedical Research 2016; 2:8-14.

13. Qiao, H., et al. Orally delivered polycurcumin responsive to bacterial reduction for targeted therapy of inflammatory bowel disease. Drug Delivery 2017; 24:233-242. contexts. Yang et al., for instance, argue the efficacy of the medicine when it comes to early detection of tumors. Early detection of tumors would spell better prognosis for the patient. Here nanotechnology is not only playing the role of a treatment plan, but it is also playing the role of a proactive agent when it comes to treating cancer. Similarly, as Sun et al. highlight, the blood-brain BBB barrier that can be crossed by nanotechnology helps deliver psychosis drugs in a better way to patients without affecting their other normal cells. Targeted deliveries and improved and overall treatment plans improve as well.

\section{Conclusion}

Multiple drugs are used with one vehicle such as a nanodrug delivery method. Nanotechnology holds many advantages for chemotherapeutic interventions and although scientists have produced various treatments of drugs with targeted delivery options, none are as receptive as the combined nanotechnology and peptide options. It is recommended that more research can be carried out on ligand structures and how it could help in targeted drug delivery.

14. Liu, F., Sun, Y., Kang, C. \& Zhu, H. Pegylated Drug Delivery Systems: From Design to Biomedical Applications. Nano LIFE 2016; 6:1642002.

15. Sun, Y., Kang, C., Yao, Z., Liu, F. \& Zhou, Y. Peptide-Based Ligand for Active Delivery of Liposomal Doxorubicin. Nano Life 2016; 6:1642004.

16. Fan, S., Huang, K., Ai, R., Wang, M. \& Wang, W. Predicting CpG methylation levels by integrating Infinium HumanMethylation450 BeadChip array data. Genomics 2016; 107:132-137.

17. Waller, A.P., et al. GLUT12 functions as a basal and insulinindependent glucose transporter in the heart. Biochimica et Biophysica Acta (BBA)-Molecular Basis of Disease 2013; 1832:121-127.

18. Li, Q., et al. Identification by shape-based virtual screening and evaluation of new tyrosinase inhibitors. PeerJ 2018; 6:e4206.

19. Chen, Y., et al. Identification of 4-aminoquinoline core for the design of new cholinesterase inhibitors. PeerJ 4, e2140 (2016).

20. Kang, C. \& Hu, K. Impact of hypoxia in the expression and regulation of the TASK-1 potassium channel in cardiac myocytes. The FASEB Journal 2016; 30;lb598-lb598.

21. Kang, C. Ion channels, protein kinase $C$ and caveolae in cardioprotection, (The Ohio State University, 2015).

22. Yung, B.C., et al. Lipid nanoparticles composed of quaternary amine-tertiary amine cationic lipid combination (QTsome) for therapeutic delivery of AntimiR-21 for lung cancer. Molecular pharmaceutics 2016; 13:653-662.

23. Cheng, X., et al. Lipid Nanoparticles Loaded with an Antisense Oligonucleotide Gapmer Against Bcl-2 for Treatment of Lung Cancer. Pharmaceutical research 2017; 34:310-320.

24. Song, L., et al. Crocetin inhibits lipopolysaccharide-induced inflammatory response in human umbilical vein endothelial cells. Cellular Physiology and Biochemistry 2016; 40:443-452.

25. Sun, Y., Kang, C., Liu, F. \& Song, L. Delivery of antipsychotics with nanoparticles. Drug Development Research 2016; 77:393399.

26. Kang, C., et al. Delivery of nanoparticles for treatment of brain tumor. Current Drug Metabolism 2016; 17:745-754.

27. Xue, X., et al. Discovery of novel inhibitors disrupting HIF$1 \alpha /$ von Hippel-Lindau interaction through shape-based screening and cascade docking. PeerJ 2016; 4:e2757. 
28. Hersch, S.J., et al. Divergent protein motifs direct elongation factor P-mediated translational regulation in Salmonella enterica and Escherichia coli. MBio 2013; 4:e00180-00113.

29. Shuhong, X., et al. Dynamic expression of AQP4 in early stageof ischemia/reperfusion rats and cerebral edema. Chinese Pharmacological Bulletin 2016; 32:1433-1441.

30. Peng, J., et al. Enhanced Liver Regeneration After Partial Hepatectomy in Sterol Regulatory Element-Binding Protein (SREBP)-1c-Null Mice is Associated with Increased Hepatocellular Cholesterol Availability. Cellular Physiology and Biochemistry 2018; 47:784-799.

31. Yang, Z., et al. Functional exosome-mimic for delivery of siRNA to cancer: in vitro and in vivo evaluation. Journal of Controlled Release 2016; 243:160-171.

32. Kang, C., Hernandez, V.A. \& Hu, K. Functional interaction of the two-pore domain potassium channel TASK-1 and caveolin-3.
Biochimica et Biophysica Acta (BBA)-Molecular Cell Research 2017; 1864:1537-1544.

33. Kang, C., Qin, J., Osei, W. \& Hu, K. Age-dependent Mitochondrial Targeting Of Protein Kinase $\mathrm{C}$ Epsilon In Cardioprotection. The FASEB Journal (2017).

34. Han, R., Sun, Y., Kang, C., Sun, H. \& Wei, W. Amphiphilic dendritic nanomicelle-mediated co-delivery of 5-fluorouracil and doxorubicin for enhanced therapeutic efficacy. Journal of Drug Targeting 2017; 25:140-148.

35. Sun, Y., et al. Co-delivery of dual-drugs with nanoparticle to overcome multidrug resistance. European Journal of BioMedical Research 2016; 2:12-18.

36. Liu, F., Sun, Y. \& Kang, C. Controlling Amphiphilic Functional Block Copolymers' Self-Assembly: From Structure to Size. (2016). 